\title{
Material selection of polymeric composite automotive bumper beam using analytical hierarchy process
}

\begin{abstract}
Selection of materials, as an area of design research, has been under considerable interest over the years. Materials selection is one of the most important activities in the product development process. Inappropriate decision of materials can cause the product to be reproduced or remanufactured. To avoid this circumstance, one of the useful tools that can be employed in determining the most appropriate material is analytical hierarchy process (AHP). To illustrate the application of AHP, six different types of composite materials were considered. The most appropriate one for suitability of use in manufacturing automotive bumper beam was determined by considering eight main selection factors and 12 sub-factors. The AHP analysis reveals that the glass fibre epoxy is the most appropriate material because it has the highest value $(25.7 \%$, mass fraction) compared with other materials. The final material is obtained by performing six different scenarios of the sensitivity analysis. It is proved that glass fibre epoxy is the most optimum decision.
\end{abstract}

Keyword: composite, polymer matrix, bumper beam, analytical hierarchy process 